\title{
Designing clinical-grade integrated strategies for the downstream processing of human mesenchymal stem cells
}

\author{
Bárbara Cunha ${ }^{1,2}$, Margarida Serra ${ }^{1,2}$, Cristina Peixoto ${ }^{1,2}$, Marta Silva ${ }^{1,2}$, Manuel Carrondo ${ }^{2,3}$, Paula Alves , $^{1 *}$ \\ From 23rd European Society for Animal Cell Technology (ESACT) Meeting: Better Cells for Better Health \\ Lille, France. 23-26 June 2013
}

\section{Background}

During the past decade, human stem cells have been the focus of an increased interest due to their potential in clinical applications, as a therapeutic alternative for several diseases. Within this context, human mesenchymal stem cells (hMSCs) have gained special attention due to their immune-modulatory characteristics, as well as in secreting bioactive molecules with anti-inflammatory and regenerative features [1].

In order to face the high demands of hMSCs (from $10^{5}$ to $10^{9}$ cells per patient) [2] to be used in therapies, the establishment of robust manufacturing platforms that can ensure the efficient production, purification and formulation of stem cell-based products is still a challenge. Although substantial efforts have been performed on the development of clinical-grade bioprocesses for the expansion of hMSCs in microcarrier-based stirred culture systems, the incorporation of downstream strategies that assure efficient cell-bead separation and consequent hMSC concentration (i.e. volume reduction) and washing is required to deliver safe hMSCs to the clinic $[3,4]$.

Therefore, the main aim of this work was the design of integrated methodologies (filtration and membrane technology approaches) [5] for the robust and clinicalgrade downstream processing of hMSC.

\section{Materials and methods}

Cell culture: hMSCs (STEMCELL Technologies ${ }^{\mathrm{TM}}$ ) were cultivated in IMDM supplemented with $10 \%$ of fetal bovine serum (FBS) or in MesenCult ${ }^{\mathbb{R}}$-XF Medium (STEMCELL Technologies ${ }^{\mathrm{TM}}$ ) supplemented with $2 \mathrm{mM}$

\footnotetext{
* Correspondence: marques@itqb.unl.pt

'Instituto de Tecnologia Química e Biológica, Universidade Nova de Lisboa, Av. da República, 2780-157 Oeiras, Portugal

Full list of author information is available at the end of the article
}

L-glutamine (Life Sciences) at $37^{\circ} \mathrm{C}$ in a humidified atmosphere of $5 \% \mathrm{CO}_{2}$, according to manufacture recommendations. These cells were routinely propagated in static conditions (T-flasks) or on microcarriers (SoloHill Engineering, Inc) using stirred culture systems (spinner vessels and bioreactors). Cell concentration and viability were determined by counting the cells in a hemacytometer using the standard trypan blue exclusion method.

Cell characterization and quality control tests: Standard procedures for the analysis of cell surface markers (CD90, CD73, CD45, CD34) using flow cytometry tools, as well as cell-based assays for the evaluation of cell proliferation capacity (CFU assay - colony-forming unit) and differentiation potential (differentiation into osteoblasts and adipocytes) were performed, following the manufacturer's recommendations.

Downstream processing: After harvesting, the microcarriers were removed from the cell suspension using nylon filters (Millipore) with different pore sizes (100, 80 and $30 \mu \mathrm{m})$. The clarified cell-based materials were concentrated by tangential flow filtration (TFF) using polysulfone hollow-fiber cartridges with $0.45 \mu \mathrm{m}$ pore size.

\section{Results}

Over the past years, as scale-up platforms for the biomanufacturing of hMSCs become robust enough to yield high cell quantities to support cell-based therapies, culture media supplemented with FBS are becoming less used. This requirement is in line with what is advised by regulatory agencies, due to the main drawbacks associated to the use of FBS, such as the variability between different lots and suppliers and the risk of contamination with animal pathogens, which may trigger an immune response upon MSC therapy [5]. Within this context, large efforts have 
been made towards the development of serum- and xenofree culture medium formulations for the expansion of hMSC. Thus, on a first approach, we evaluated the feasibility of propagating hMSCs in a serum-and xeno-free culture medium, the MesenCult ${ }^{\mathbb{R}}-\mathrm{XF}$ medium, and further compared cell growth profile with standard medium formulation (e.g. IMDM + 10\% FBS). Our results showed that, hMSC can be successfully expanded in MesenCult ${ }^{\mathbb{R}}$ XF medium, presenting a constant population doubling length (PDL) of approximately 2 in each cell passage and a cumulative PDL of 12.5 in a total of 42 days (Figure 1A). Moreover, hMSCs showed an accelerated cell growth and increased lifespan when compared to the hMSC cultivated in standard culture medium supplemented with FBS where hMSCs presented limited proliferation capacity (Figure 1A). This was an expected outcome since MesenCult ${ }^{\mathbb{B}}$-XF medium was design to enhance hMSC expansion from primary human bone marrow and cultured-expanded cells, leading to long-term cultures. It is important to mention that hMSCs maintained their characteristics after expansion in MesenCult ${ }^{\circledR}$-XF medium, namely immunophenotype, proliferation capacity and multipotency (results not shown).

After the expansion of hMSCs in microcarrier-based stirred culture systems, different downstream strategies were evaluated for the purification of hMSCs. First, the clarification step was carried out to remove the microcarriers from the cell suspension. For this purpose, nylon filters were used and the effect of the mesh pore size on cell recovery yields and viability was evaluated. Our results showed that nylon is a suitable material for the clarification step since it ensured efficient removal of microcarriers (no beads were detected after filtration processing) without compromising cell viability (Figure 1B). Moreover, we demonstrated that higher mesh pore sizes yielded higher cell recoveries (Figure 1B).

For the cell concentration and volume reduction step, preliminary experiments were performed with human foreskin fibroblasts (Figure 1C). With this cellular system, a concentration factor (in volume) of 10 times was successfully achieved using TFF processes, yielding 70-80\% of recovered cells with high viabilities (Figure 1C).
A

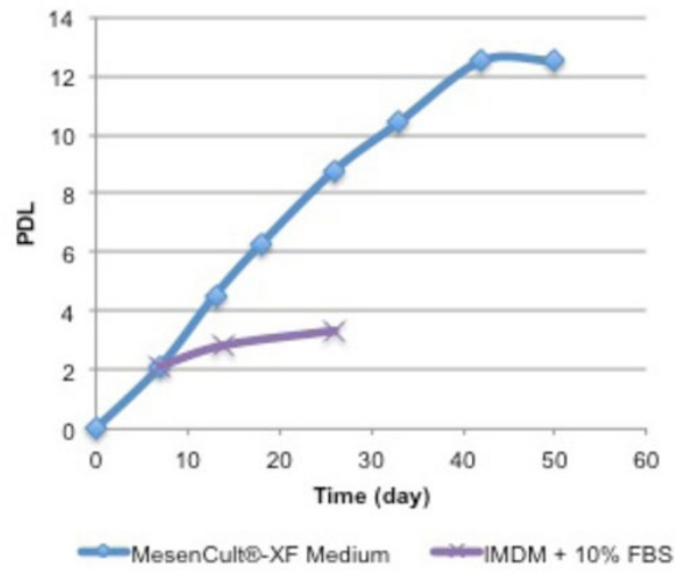

B

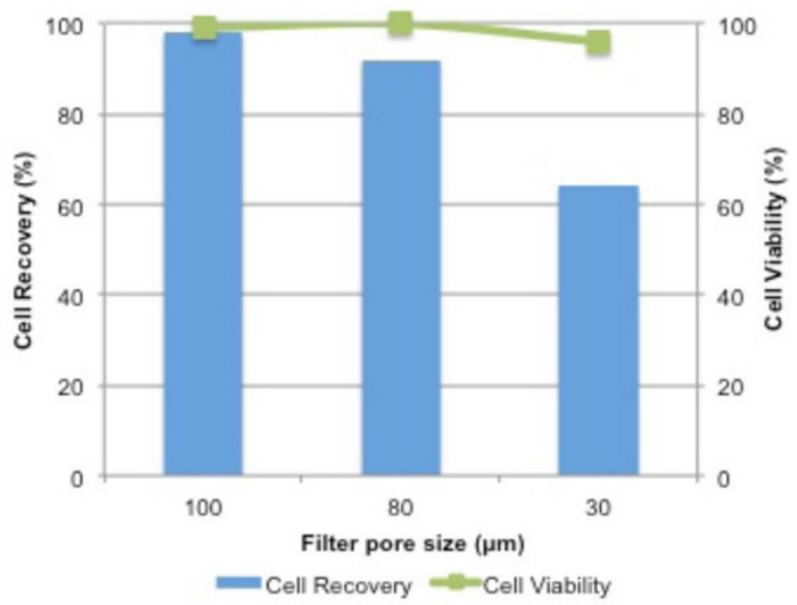

Human Foreskin Human Mesenchymal Fibroblasts

\begin{tabular}{|c|c|c|}
\hline C & Up to 10 & 2 \\
\hline Cell recovery yield (\%) & $70-80$ & 96 \\
\hline Cell viability (\%) & $>98$ & 98 \\
\hline
\end{tabular}

Figure 1 Up- and down- stream processing of hMSCs. A) Growth profile of hMSC culture; profile of cumulative PDLs of hMSC cultured in MesenCult ${ }^{\mathbb{R}}$-XF (blue line) or in IMDM $+10 \%$ FBS (purple line) medium along culture time. B) Microcarriers' removal using nylon filters with different pore sizes (100,80 and $30 \mu \mathrm{m}$ ). The (blue) bars represent the cell recovery yields, while the (green) line represents cell viability. C) Major outcomes achieved after TFF processing of human foreskin fibroblasts and hMSCs. 
Process validation with hMSCs is ongoing but first results were encouraging since we were able to concentrate 2 times hMSCs while ensuring high cell recovery yields (96\%) and viabilities (98\%) (Figure 1C). In addition, hMSCs maintained their immunophenotype, as well as their proliferation capacity and multilineage differentiation potential at the end of all steps of the downstream process (results not shown).

\section{Conclusions}

While upstream technologies mature to meet the increasing demand of hMSCs, biomanufacturing bottlenecks are now shifting towards the downstream processing of stem cells. This work shows our first approach to tackle such bottlenecks. More specifically, we demonstrate that standard filtration techniques and TFF systems are suitable and robust approaches for the downstream processing of hMSCs. Using these strategies we were able to ensure efficient removal of the major impurities of the cellular suspension (microcarriers) and further concentrate cellbased products up to 10 times without compromising their viability and quality. However, further improvements in cell concentration and polishing steps are still required. Nonetheless, this work provides important insights towards the establishment of robust and clinicalgrade bioprocesses for the purification of hMSCs to be integrated and applied in the biomanufacturing of cellbased therapies.

\section{Acknowledgements}

The authors acknowledge the NanoGene project (EuroNanoMed ERA-Net initiative) and the project EXPL/BBB-EBI/1003/2012 - "Development of a scalable strategy for stem cells purification funded by Fundação para a Ciência e Tecnologia (FCT) for financial support, as well as MIT-Portugal program and FCT for the grant SFRH/BD/51940/2012.

\section{Authors' details}

'Instituto de Tecnologia Química e Biológica, Universidade Nova de Lisboa, Av. da República, 2780-157 Oeiras, Portugal. ${ }^{2}$ iBET, Instituto de Biologia Experimental e Tecnológica, Apartado 12, 2780-901 Oeiras, Portugal.

${ }^{3}$ Departamento de Química, Faculdade de Ciências e Tecnologia,

Universidade Nova de Lisboa, 2829-516 Monte da Caparica, Portugal.

Published: 4 December 2013

\section{References}

1. Patel A, Genovese J: Potential clinical applications of adult human mesenchymal stem cell (Prochymal ${ }^{\oplus}$ ) therapy. Stem Cells and Cloning: Advances and Applications 2011, 4:61-72.

2. Chen A, Reuveny S, Oh S: Application of human mesenchymal and pluripotent stem cell microcarrier cultures in cellular therapy: Achievements and future direction. Biotechnology Advances 2013.

3. Serra M, Brito C, Correia C, Alves PM: Process engineering of human pluripotent stem cells for clinical application. Trends in Biotechnol 2012, 30:350-359.

4. Pattasseril J, Varadaraju H, Lock L, Rowley J: Downstream Technology Landscape for Large-Scale Therapeutic Cell Processing. BioProcess International 2013, 11:46-52.

5. Peixoto C, Ferreira T, Sousa M, Carrondo MJT, Alves PM: Towards purification of adenoviral vectors based on membrane technology. Biotechnol Prog 2008, 24:1290-1296.
6. Spees J, Gregory C, Singh H, Tucker H, Peister A, Lynch P, Hsu S, Smith J, Prockop D: Internalized antigens must be removed to prepare hypoimmunogenic mesenchymal stem cells for cell and gene therapy. Mol Ther 2004, 9:747-756.

doi:10.1186/1753-6561-7-S6-P103

Cite this article as: Cunha et al:: Designing clinical-grade integrated strategies for the downstream processing of human mesenchymal stem cells. BMC Proceedings 2013 7(Suppl 6):P103.

\section{Submit your next manuscript to BioMed Central and take full advantage of:}

- Convenient online submission

- Thorough peer review

- No space constraints or color figure charges

- Immediate publication on acceptance

- Inclusion in PubMed, CAS, Scopus and Google Scholar

- Research which is freely available for redistribution 